\title{
EDITORIAL
}

\section{La ratificación del Convenio Marco para el Control del Tabaco}

E 1 Convenio Marco para el Control del Tabaco ${ }^{1}$ (CMCT) es el primer tratado multilateral iniciado por la Asamblea Mundial de la Salud. Esencialmente se trata de una convocatoria a la cooperación internacional basada en la suficiente evidencia científica sobre los daños que el tabaco causa a la salud. Recientemente, el 28 de mayo de 2004, México ratificó dicho convenio. El CMCT se aprobó unánimemente hace un año; le sucedió el proceso de firmas, que lento, pero sostenido, logró que poco más de la mitad de los miembros de la Organización Mundial de la Salud ya hayan firmado. Mayormente, en América Latina y El Caribe las legislaciones sobre el control del tabaco no han sido muy rigurosas ${ }^{2} y$, hasta ahora, sólo México ha ratificado dicho documento. Al escribir este Editorial todavía son menos de 20 países los que han dado el paso comprometido de la ratificación.

A diferencia de otros eventos donde el punto culminante es lo más publicitado, la ratificación del CMCT es sólo el inicio e implica comprometerse a recorrer un camino. No hay margen para fechas emblemáticas, pues se trata de algo a cumplir cada día. Igualmente, la adherencia a este Convenio no nos será dada de una vez por todas, al contrario, es una actitud siempre susceptible de ser abandonada. El CMCT está en muchas acciones implementadas integralmente y el descuido de unas no se compensa con el reforzamiento de otras. Ratificarlo implica compromiso, y el compromiso es coherencia. La coherencia en el control del tabaco son las medidas de reducción de la demanda (Artículos 6 al 14), de la oferta (Artículos 15 al 17) y de los daños, con el objeto de mejorar la salud de la población -eliminando o disminuyendo su consumo de productos de tabaco y su exposición al humo de éste (Artículo 1d).

Ubicar a México ante el Convenio que acaba de ratificar, implica necesariamente revisar cómo estamos en los puntos clave que dicho documento plantea, como son: lograr reducir el consumo (Artículo 3); pro- tección de los no fumadores (Artículo 8); revelar los componentes del humo de tabaco (Artículos 9 y 10); eliminar los términos engañosos que usa la industria (Artículo 11); la prohibición absoluta de la publicidad y el patrocinio (Artículo 13); así como garantizar la no venta a menores de edad y la distribución gratuita de cigarros (Artículo 16). Si convenio marco significa acuerdo de referencias o marcas pactadas, en ambos casos es necesario evaluar. Evaluar sistemáticamente para saber cómo vamos en el cumplimiento de lo aprobado, lo firmado y lo ratificado.

De la reciente aplicación de la Encuesta sobre Tabaquismo en Jóvenes, México 2003, ${ }^{3}$ sabemos que la mitad (51.1\%) de los estudiantes de secundaria ha probado el cigarro y que $19.9 \%$ fuma actualmente, sin diferencias entre hombres y mujeres. El consumo de tabaco en edades tan jóvenes se asocia con un bajo desempeño escolar ${ }^{4}$ y como droga de inicio al consumo de otras sustancias ilegales. ${ }^{5}$ Además, los adolescentes están muy expuestos al humo del tabaco de otros, tanto en su casas $(45.7 \%)$, como en lugares públicos (54.5\%). Lo anterior, a pesar de que $74.4 \%$ cree que el humo de otros les es dañino. La publicidad de cigarros sigue siendo intensa, porque $73.9 \%$ de los estudiantes de 13 a 15 años, la ha visto en revistas recientemente y $93.4 \%$ refiere haber visto actores fumando en programas de cine y de televisión. Una realidad alarmante es que 37.0\% de esos fumadores tan jóvenes compra sus cigarros en las tiendas y que a $61.8 \%$ de ellos no se les negó la venta en el último mes, a pesar de ser menores de edad. Asimismo, a 16.9\% de los estudiantes fumadores los representantes de la industria tabacalera les han ofrecido cigarros gratis.

Para combatir la realidad antes descrita hay que reconocer inequívocamente al tabaco como dañino para la salud, ese es el motor del CMCT. La industria tabacalera y el sector salud son antagónicos, como también lo son sus intereses y prioridades. Quien consuma una cajetilla diaria, irremediablemente acabará enferman- 
do y eventualmente morirá prematuramente. El costo del tratamiento médico durante un año de cualquier enfermedad atribuible al consumo de tabaco supera exponencialmente cualquier contribución hecha por un comprador de productos del tabaco en igual período.

La medida más efectiva ${ }^{6}$ para reducir el consumo es la elevación de los precios del cigarro a través de impuestos (Artículo 6). Nada como lograr que la gente no fume, o que fume menos. A eso es a lo que llama básicamente el CMCT. El esquema impositivo vigente, impulsado por el actual Secretario de Salud, muestra su efecto positivo en la reducción de la proporción de hogares con gasto en tabaco, según la Encuesta Nacional de Ingreso y Gasto de los Hogares. Al mismo tiempo, no ha disminuido la recaudación fiscal, sino todo lo contrario, como lo ilustran las cifras de la Secretaría de Hacienda y Crédito Público. En México, ya se ha documentado ${ }^{7}$ que el precio y el ingreso son los determinantes más importantes del gasto en tabaco, por lo que el Instituto Nacional de Salud Pública ha impulsado sostenidamente la aplicación de impuestos como la principal medida de reducción de la demanda.

Desde hace décadas se conoce que el consumo de tabaco es causa de muertes prematuras por más de 20 enfermedades, entre ellas diferentes tipos de cáncer y de enfermedades respiratorias y cardiovasculares. Recientemente se han documentado ${ }^{8}$ nuevos vínculos causales para el cáncer de estómago, cáncer cérvico uterino, de páncreas y de riñón; además, con aneurisma aórtico abdominal, leucemia mieloide aguda, neumonía, cataratas y periodontitis.

El texto del Convenio Marco es una amenaza cierta para todo el que hace del tabaco su negocio. Por ello, uno de los retos medulares que tenemos por delante al haberlo ratificado es el que ya se advierte en el párrafo 19 del Preámbulo: "(...) mantener la vigilancia ante cualquier intento de la industria del tabaco de socavar o desvirtuar las actividades de control del tabaco, y la necesidad de estar informados de las actuaciones de la industria del tabaco que afecten negativamente a las actividades de control del tabaco."

En ese mismo sentido deberíamos enfrentar como una de las obligaciones generales la que aparece en el punto 3 del Artículo 5: "A la hora de establecer y aplicar sus políticas de salud pública relativas al control del tabaco, las Partes actuarán de una manera que proteja dichas políticas contra los intereses comerciales y otros intereses creados por la industria tabacalera, de conformidad con la legislación nacional."

Ahora que se ha ratificado el CMCT, las autoridades, los grupos de investigación y la sociedad en su conjunto deben apoyar y vigilar el cumplimiento de las acciones que desalienten el consumo de tabaco, todos unidos bajo el sólido argumento de que la salud es un derecho humano fundamental.

Maestro Raydel Valdés Salgado*

\section{Referencias}

1. O rganización Mundial de la Salud. Convenio Marco para el Control del Tabaco. G inebra: Suiza; 2003. D isponible en: http://www.who.int/gb/ ebwha/pdf files/W HA56/sa56r1.pdf [6 junio 2004].

2.Valdés-Sälgado R, Hernández-A vila M, Sepúlveda-A mor J. El consumo de tabaco en la región americana: elementos para un programa de acción. Salud Publica Mex 2002;44,supl 1:S125-S135.

3.Valdés-Salgado R, Meneses-González F, Lazcano-Ponce EC, Hernández-Ramos MI, Hernández-Avila M. Encuesta sobre tabaquismo en jóvenes, México 2003. Cuernavaca: Instituto $\mathrm{N}$ acional de Salud Pública; 2004.

4.A rillo-Santillán E, Fernández E, Hernández-Avila M,Tapia-U ribe M, Cruz-Valdez A, Lazcano-Ponce EC. Prevalencia de tabaquismo y bajo desempeño escolar, en estudiantes de 11 a 24 años de edad del estado de Morelos, México. Salud Publica Mex 2002;44,supl 1:S54-S66. 5. Medina-Mora ME, Peña-C orona MP, Cravioto P,Villatoro J, Kuri P. D el tabaco al uso de otras drogas: iel uso temprano de tabaco aumenta la probabilidad de usar otras drogas? Salud Publica Mex 2002;44,supl 1:S109-S115.

6. Jha P, Chaloupka F. Ed. Tobacco control in developing countries. N ew York (N Y): 0 xford University Press; 2000.

7. Sesma-Vázquez S, C ampuzano-Rincón JC, C arreón-RodríguezVG, Knaul F, López-A ntuñano FJ, Hernández-Avila M. El comportamiento de la demanda de tabaco en México: 1992-1998. Salud Publica Mex 2002;44,supl 1:S82-592.

8. US D epartment of Health and Human Services. The health consequences of smoking:A Report of the Surgeon General. Atlanta (GA): US D epartment of Health and Human Services. Centers for Disease Control and Prevention. $N$ ational $C$ enter for Chronic D isease Prevention and Health Promotion, 0 ffice on Smoking and Health; 2004.

\footnotetext{
* Jefe del Departamento de Investigación sobre Tabaco. Instituto Nacional de Salud Pública. Cuernavaca, Morelos, México. Correo electrónico: rayvs@insp.mx
} 